\title{
Utility-based Resource Allocation for Interference Limited OFDMA Cooperative Relay Networks
}

\author{
Nidhal Odeh ${ }^{\mathrm{a}}$, Mehran Abolhasan ${ }^{\mathrm{a}}$, Farzad Safaei ${ }^{\mathrm{b}}$, Daniel R. Franklin ${ }^{\mathrm{a}}$, \\ Guoqiang $\mathrm{MaO}^{\mathrm{a}}$ \\ ${ }^{a}$ School of Computing and Communications, University of Technology Sydney, Broadway \\ NSW 200\%, Australia \\ ${ }^{b}$ ICTR Institute, Faculty of Informatics, University of Wollongong, Australia
}

\begin{abstract}
This paper proposes a utility-based resource allocation algorithm for the uplink OFDMA Inter-cell Interference (ICI) limited cooperative relay network. Full channel state information (CSI) is assumed to be available at the resource controller at initial stage, then the work is extended to consider more realistic assumption, i.e., only partial channel state information (PCSI) is available. The proposed algorithm aims to maximize the total system utility while simultaneously satisfying the individual user's minimum data rate requirements. In the proposed algorithm, relay selection is initially performed based on the consideration of ICI. Then, subcarrier allocation is performed to achieve maximum utility assuming equal power allocation. Finally, based on the amount of ICI, a modified water-filling power distribution algorithm is proposed and used to optimize the per-carrier power allocation across the allocated set of subcarriers. The results show that, compared to conventional algorithms, the proposed algorithm significantly improves system performance in terms of total sum data rate, outage probability and fairness.
\end{abstract}

Email addresses: Nidhal.0deh@student.uts.edu.au (Nidhal Odeh), Mehran.Abolhasan@uts.edu.au (Mehran Abolhasan), farzad@uow.edu.au (Farzad Safaei), Daniel.Franklin@uts.edu.au (Daniel R. Franklin), Guoqiang.Mao@uts.edu.au (Guoqiang Mao) 


\section{Introduction}

Cooperative communications is emerging as an important area within the field of wireless communication systems. The fundamental idea is that intermediary nodes, called relay stations (RSs), who are neither the data source nor the destination, are used to assist in communications between senders and receivers. In order to maximize their performance, networks which employ $\mathrm{RSs}$ require a new resource allocation and optimization technique, which takes the RSs into account as a new resource.

Several resource allocation algorithms have been proposed for the purpose of maximizing the average data rates under power constraints [3], [18], [9],[26] and [24]. The authors of [17], [21] presented resource allocation algorithms which aim to optimize the distribution of resources between users while maintaining a satisfactory degree of fairness amongst them. In fact, there exists a trade-off between fairness and capacity; the imposition of fairness constraints on the optimization problem will often degrade aggregate system capacity. Utility-based resource allocation algorithms have been developed to balance the trade-off between subscribers fairness, system capacity and other performance metrics such as latency [12], [22].

The authors of [5] proposed a resource allocation scheme with adaptive priority thresholds. The proposed algorithm balances the trade-off between the minimum data rate requirement satisfaction and the capacity. Yen et al proposed a different utility-based throughput maximization and complexityreduction scheduling scheme [20], which allocates subcarriers, antenna sequence, and modulation order to multimedia users for the purpose of maximizing the total capacity under the minimum data rate requirements constraints. In both of these papers, a single cell scenario has been considered only, i.e., interference from other cells is neglected. However, it is important to consider the effects of interference caused by neighboring cells (Inter-cell Interference or ICI) in the resource optimization due to its impact on the system performance [27].

Recently, Zhang et al investigated the joint uplink subchannel and power allocation problem in cognitive small cells with the presence of cross-tier interference under the assumption of imperfect channel state information (CSI) [23]. The proposed cooperative Nash bargaining resource allocation algorithm aims to maximize the achievable sum rate without compromising the outage probability and fairness among users. The work of [23] has been extended in [25] to include cotier and cross-tier interference mitigation taking 
spectrum sensing errors into account. However, in these papers cotier and/or cross-tier interference is considered as the main interference source while cochannel interference between small cells is assumed as part of the thermal noise. Additionally, these papers do not specify the relaying protocol that is used by the small cells.

Inter-cell interference have been considered in [7]. This paper assumes cooperation between neighboring base stations (BSs). However, the information exchange across different cells introduces an overhead on the backbone network.

Most of the existing literature considers the minimum data rate requirements and capacity trade-off and aims to balance this trade-off sequentially [13], [15]. This approach implies that priority will always be given to the subscriber with the highest instantaneous rate requirements. This may lead to excessive use of available resources by a single subscriber if that subscriber is in a deep fade. Thus, the aggregate capacity is degraded.

In addition to that, another limitation was observed in previous related papers which relates to the availability of channel state information (CSI). It can be seen the proposed algorithms assume that the CSI is fully and accurately available at the resource controller. However, this assumption is unrealistic due to channel estimation error and the feedback delay.

Motivated by the above review, in this paper we study the resource allocation and optimization for inter-cell interference limited OFDMA-based cooperative relay network with full and partial CSI available at the resource controller.

More specifically, this paper proposes a utility-based resource allocation, in which the current minimum data rate requirements of every user and the impact of the allocation of every subcarrier on the total capacity will be jointly considered to dynamically update the selection priority. Furthermore, the CSI is assumed to be available at the resource controller at initial stage, then the work is extended to consider more realistic assumption, i.e., only partial channel state information (PCSI) is available.

The proposed utility-based resource allocation algorithm is divided into three stages: relay selection, subcarrier allocation and then power allocation. The proposed algorithm aims to maximize the total achievable network data rate. ICI and fairness issues are taken into account during the resource allocation.

The contributions and novelties of this paper are summarized in the following. 
- New utility and performance degradation functions are developed and incorporated into the proposed utility-based resource allocation algorithm in order to optimize the available resources, such that the aggregate data rate is maximized while meeting the constraints on the minumum data rate requirements and fair resources distribution.

- A modified water-filling power allocation algorithm has been developed by which the available power is allocated across subcarriers based on the amount of ICI on each subcarrier such that subcarriers with high ICI are avoided.

- The system performance has been evaluated under realistic assumptions and considerations, such as partial channel state information (PCSI), presence of inter-cell interference (ICI) and fairness requirements.

The remainder of this paper is organised as follows: Section 2 presents the system model. Section 3 defines and models the utility and performance degradation functions. Section 4 formulates the optimization problem. Section 5 presents the proposed utility-based resource allocation algorithms, while the proposed ICI-based water-filling algorithm is presented in Section 6. Section 7 presents the partial channel state information model, while in Section 8, performance of the proposed algoithms is evaluated by simulations. finally, Section 9 concludes the paper.

\section{System Model}

This paper considers a multiple-cell scenario as shown in Figure 1. The subscriber stations, amplify and forward (AF) relay stations and destination are denoted as $S, R$, and $D$ respectively. The cell under consideration receives an ICI from the interference sources $I$ of neighboring cells. This ICI interference is received from neighboring cells with varying signal strength levels depending mainly on the distances between each one of the interference nodes and the node under consideration. The interference on the $r^{\text {th }}$ relay station from the $i^{\text {th }}$ interference node denoted as $I_{i, r}$ and can be expressed as [4]:

$$
I_{i, r}=H_{i, r} L\left(d_{i, r}\right) p_{i}
$$

where, $p_{i}, L\left(d_{i, r}\right)$ and $H_{i, r}$ are the transmission power at the interference node, the pathloss and the instant channel respectively. Parameter $d_{i, r}$ 
represents the distance between the $i^{\text {th }}$ interfering node and the $r^{\text {th }}$ node of interest which is variable parameter as different interfering nodes are located at different distances from the node of interest .

Moreover, the available bandwidth is divided into $(N)$ subcarriers and are available at the destination. Denote the set of source nodes, relay nodes, interference nodes and subcarriers as $\mathcal{S}=\{1, \ldots, s, \ldots, S\}, \mathcal{R}=\{1, \ldots, r, \ldots, R\}$, $\mathcal{I}=\{1, \ldots, i, \ldots, I\}$ and $\mathcal{N}=\{1, \ldots, n, \ldots, N\}$ respectively.

Furthermore, we assume that the $\mathrm{RS}$ of the neighboring cells transmits data with equal transmission power and using the same transmission frequency (i.e., full frequency reuse), and therefore, cause ICI on the cell under consideration. Thus, the serving BS has knowledge about the transmission power of the $i^{\text {th }}$ interference source $\left(P_{i}\right)$ and therefore, the average power across each interfering subcarrier $\left(n^{\text {th }}\right.$ subcarrier) is given by $p_{i}^{n}=P_{i} / N, i=1, \ldots, I$

The transmission is performed in two time frames. In the first time frame $T_{1}$, the subscriber station $s \in \mathcal{S}$ transmits the signal over a number of subcarriers with a transmission power across each subcarrier of $p_{s}^{n}$. The total transsmision power by each source $p_{s}$ using all subcarriers should not exceed the predetermined total transmission power $P_{T}$. The $r^{\text {th }}$ relay station $r \in \mathcal{R}$ receive the transmitted signal with ICI from neighboring cells. The received signal-to-interference-plus-noise ratio $(S I N R)$ at the $r^{\text {th }}$ relay station is given by:

$$
S I N R_{r}^{n}=\frac{\left|H_{s, r}^{n}\right|^{2} p_{s}^{n}}{\sigma_{r}^{2}+\sum_{i=1}^{I}\left|H_{i, r}^{n}\right|^{2} L\left(d_{i, r}\right) p_{i}^{n}}
$$

where $H_{s, r}^{n}$ denotes the instant channel between the $s^{\text {th }}$ subscriber station and $r^{\text {th }}$ relay station over the $n^{\text {th }}$ subcarrier, $H_{i, r}^{n}$ denotes the instant channel between the $i^{\text {th }}$ interfering cell and $r^{\text {th }}$ relay station over the $n^{\text {th }}$ subcarrier and $\sigma_{r}^{2}$ denotes the variance of the additive white Gaussian noise (AWGN) at the $r^{\text {th }}$ relay station.

Similarly, the received SINR at the destination from the direct link is given by:

$$
S I N R_{s, d}^{n}=\frac{\left|H_{s, d}^{n}\right|^{2} p_{s}^{n}}{\sigma_{d}^{2}+\sum_{i=1}^{I}\left|H_{i, d}^{n}\right|^{2} L\left(d_{i, d}\right) p_{i}^{n}}
$$

where $H_{s, d}^{n}$ denotes the instant channel between the $s^{\text {th }}$ subscriber station and the destination $d$ over the $n^{\text {th }}$ subcarrier, $H_{i, d}^{n}$ denotes the instant 
channel between the $i^{\text {th }}$ interfering cell and the destination $d$ over the $n^{\text {th }}$ subcarrier, $d_{i, d}$ represents the distance between the $i^{t h}$ interfering node and the destination $d$ and $\sigma_{d}^{2}$ denotes the variance of the AWGN at the destination $d$.

In the second time frame $T_{2}$, the RSs amplify the received signal by a factor $g_{r}$ such that the transmission power from the $r^{\text {th }}$ relay using the $n^{\text {th }}$ subcarrier equals to $p_{r}^{n}$. Similarly, The total transsmision power by each relay $p_{r}$ using all subcarriers should not exceed the predetermined total transmission power $P_{T}$. The RSs then forward the received signal to the destination, the SINR at the destination is given by:

$$
S I N R_{r, d}^{n}=\frac{\left|H_{r, d}^{n}\right|^{2} g_{r}^{2}\left|H_{s, r}^{n}\right|^{2} p_{s}^{n}}{\sum_{i=1}^{I}\left|H_{i, r}^{n}\right|^{2} L\left(d_{i, r}\right)\left|H_{r, d}^{n}\right|^{2} g_{r}^{2} p_{i}^{n}+\left|H_{r, d}^{n}\right|^{2} g_{r}^{2} \sigma_{r}^{2}+\sigma_{d}^{2}}
$$

Where, $g_{r}$ is the RS amplification factor given by [10]:

$$
\begin{gathered}
g_{r}=\sqrt{\frac{p_{r}^{n}}{p_{s}^{n}\left|H_{s, r}^{n}\right|^{2}+\sum_{i=1}^{I}\left|H_{i, r}^{n}\right|^{2} L\left(d_{i, r}\right) p_{i}^{n}+\sigma_{r}^{2}}} \\
\text { Let, } \gamma_{s, r}=\left|H_{s, r}^{n}\right|^{2} p_{s}^{n} / \sigma^{2}, \gamma_{r, d}=\left|H_{r, d}^{n}\right|^{2} p_{r}^{n} / \sigma^{2}, I_{I, r}=\sum_{i=1}^{I}\left|H_{i, r}^{n}\right|^{2} L\left(d_{i, r}\right) p_{i}^{n} / \sigma^{2}
\end{gathered}
$$
and $\sigma_{r}^{2}=\sigma_{d}^{2}=\sigma^{2}$, then by substituting (5) in (4), the SINR in (4) is written as:

$$
S I N R_{r, d}^{n}=\frac{\gamma_{s, r} \gamma_{r, d}}{I_{I, r}\left(\gamma_{r, d}+1\right)+\gamma_{s, r}+\gamma_{r, d}+1}
$$

From (6) the combined SINR at the destination $d$ from all participating relay stations is written as:

$$
S I N R_{d}^{n}=\sum_{j=1}^{J} S I N R_{j, d}^{n}=\frac{\gamma_{s, j} \gamma_{j, d}}{I_{I, j}\left(\gamma_{j, d}+1\right)+\gamma_{s, j}+\gamma_{j, d}+1}
$$

where $J$ is a variable which represents the number of relay stations that participate in forwarding the original signal to its destination $(1 \leq J \leq R)$. This variable is determined based on the outcome of the relay selection and subcarrier allocation algorithms. Users will be allocated with subcarriers and each of those subcarriers is attached to one relay station. Thus, the number of participating relay stations will depend on the outcome of which 


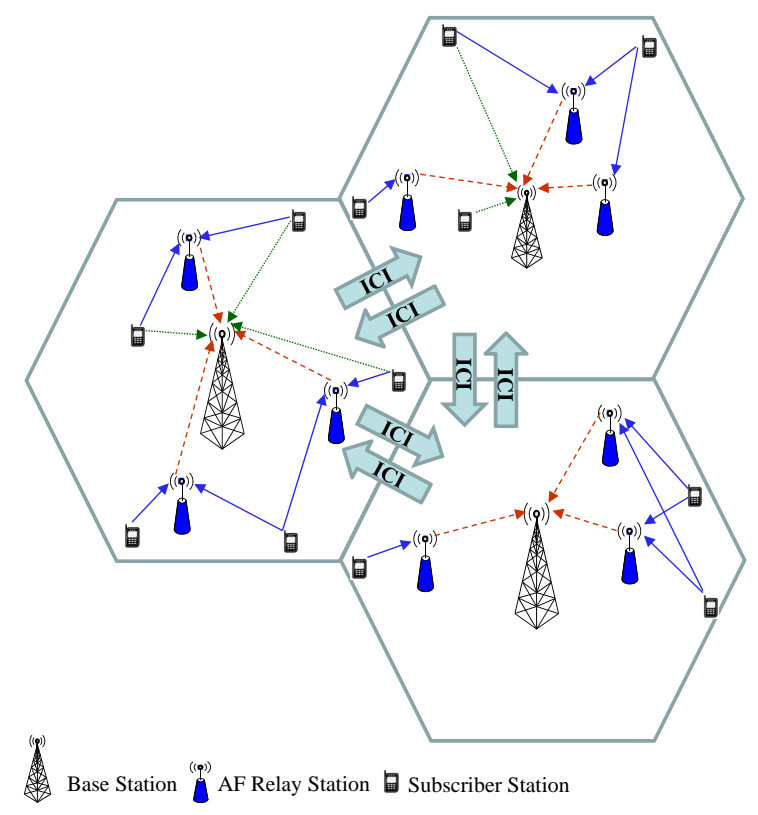

Figure 1: Multiple-Cell interference limited OFDMA-based cooperative relay system model

subcarriers one user will get allocated. These algorithms will be discussed in more details in the following sections.

\section{Utility Function Modelling and Definition}

This section models the utility function based on the system model described in Section 2 and define the objectives of this utility-based resource allocation.

The term Utility here indicates the degree of user satisfaction in terms of data rates and fairness. In fact, this paper focuses on two main objectives: guaranteeing the minimum data rate requirements and maximizing the total achievable data rate. This is achieved through relay selection, subcarrier allocation and power allocation algorithms.

Figure 2(a,b) depicts the utility function of the minimum rate satisfaction and total rate maximization objectives [11]. In the case of minimum rate satisfaction $\left(U_{R_{s_{m i n}}}\right)$, the utility is represented by a unit step function, in which a certain subscriber (the $s^{\text {th }}$ user) is considered to be satisfied when 
its minimum required data rate is achieved (i.e., $R_{s} \geq R_{s_{\min }}$ ) using certain number of subcarriers $\left(n_{s}\right)$ out of the available $\mathrm{N}$ subcarriers. This is referred to as a hard threshold.

However, the total rate maximization utility $\left(U_{R_{T}}\right)$ has no hard threshold to reach. Instead, the objective is to maximize the total sum rate. The minimum total sum data rate is the sum of the minimum rate requirements

by all subscribers $\left(\sum_{s=1}^{S} U_{R_{m i n}(s)}\right)$, as shown in Figure 2(b). Note that this is only valid when the system has a sufficient number of resource channels to satisfy all the subscribers' minimum rate requirements. Otherwise, the total sum data rate $\left(U_{R_{T}}\right)$ will be less than the sum of minimum rate requirements due to the rate un-satisfaction for some or all of the subscribers.

By contrast, inter-cell interference significantly degrades the overall system performance due to the increase in interference caused by neighboring cells. The performance degradation caused by ICI effects over the $r^{\text {th }}$ relay station is denoted by $D(r)$. In this case, the objective is to minimize this performance degradation over the relay stations through ICI-based relay selection. As shown in figure 2(c), the $r^{\text {th }}$ relay station will receive the maximum ICI when it uses the maximum number of subcarriers (i.e., $N$ ). This is due to the fact that every subcarrier will carry a fraction of the total ICI. However, since a total number of $N$ subcarriers needs to be utilised by $R$ relay stations, then every relay station will only be able to use some of the available subcarriers. Thus, the minimization of $D(r)$ here refers to carefully selecting the subcarriers to be used by each relay station, such that $D(r)$ is minimized.

In the following section, the optimization problem will be formulated based on the objective functions as defined in this section (i.e., the utility and performance degradation functions).

\section{Problem formulation}

In this section, the optimization problem is formulated for the purpose of maximizing the utility function associated with the total sum data rate (Figure 2(b)), subject to fairness and maximum power constraints. The fairness constraint is imposed to guarantee the minimum rate utility function (Figure 2(a)) to be satisfied for as many subscribers as the available resources can accommodate.

From (7), the instantaneous sum data rate utility of the $s^{\text {th }}$ subscriber on the subcarrier $n$ is given by 


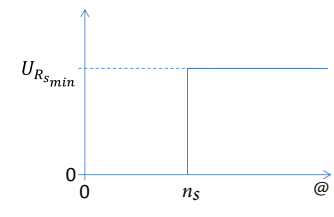

(a) Minimum Rate Satisfaction

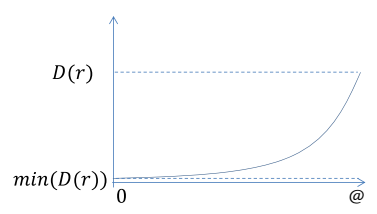

(c) Inter-cell Interference Mitigation

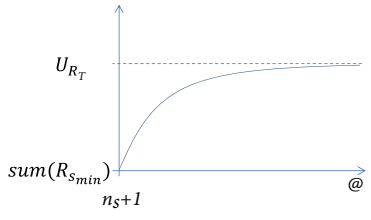

(b) Total Rate Maximizations

Figure 2: Utility and performance degradation functions

$$
U_{R_{s}^{n}}=\frac{1}{2} \log \left(1+S I N R_{d}^{n}\right)
$$

Taking into account all possible allocated subcarriers $(N)$, then using (8), the total sum data rate utility of all subscribers $(S)$ with the help of all relay stations $(R)$ can be written as:

$$
U_{R_{T}}=\sum_{r=1}^{R} \sum_{s=1}^{S} \sum_{n=1}^{N} \rho_{s, r}^{n} U_{R_{s}^{n}}
$$

where $\rho_{s, r}^{n}$ denotes the subcarrier allocation index, defined as [2]

$$
\rho_{s, r}^{n}= \begin{cases}1 & n^{\text {th }} \text { subcarrier assigned to subscriber } s \text { and relay } r \\ 0 & \text { otherwise }\end{cases}
$$

The utility-based resource allocation optimization problem can be formulated as:

$$
\max \left(U_{R_{T}}\right)
$$

subject to: 


$$
\begin{array}{r}
\rho_{s, r}^{n} \in\{0,1\}, \forall n, s, r \\
\sum_{s=1}^{S} \sum_{r=1}^{R} \rho_{s, r}^{n}=1, \forall n \\
\sum_{n=1}^{N} U_{R_{s}^{n}} \geq U_{R_{\min }(s)}, \forall s \\
\sum_{n=1}^{N} \sum_{s=1}^{S} p_{s}^{n} \leq P_{T} \\
\sum_{n=1}^{N} \sum_{r=1}^{R} p_{r}^{n} \leq P_{T} \\
p_{s}^{n} \geq 0, \forall n, s \\
p_{r}^{n} \geq 0, \forall n, r
\end{array}
$$

The constraints (11a) and (11b) indicate that each subcarrier is only allocated to a single subscriber-relay pair. Constraints (11c) guarantee the satisfaction of the minimum rate utility for all subscribers. Constraints (11d) and (11e) ensures that the transmitted power by subscribers and relay stations should not exceed the total power constraint $P_{T}$. Finally, constraints (11f) and (11g) limit the subscribers and relay stations minimum transmitted power.

The optimization problem in (10) contains both discrete and continuous variables and classified as non-convex NP-hard problem. This makes the problem intractable and computationally complex.

Conversely, the second optimization problem is to minimize the ICI effects on each relay station in order to enhance the received SINR, hence increase the data rate. This optimization problem may be formulated as:

$$
\min (D(r))
$$

subject to: 


$$
\begin{array}{r}
\rho_{r}^{n} \in\{0,1\}, \forall n, r \\
\sum_{r=1}^{R} \rho_{r}^{n}=1, \forall n \\
\sum_{r=1}^{R} \sum_{n=1}^{N} \rho_{r}^{n}=N,
\end{array}
$$

where constraints (13a) and (13b) are similar to those in (11a) and (11b) respectively, and constraint $(13 \mathrm{c})$ indicates that the available subcarriers need to be distributed among the available relay stations. In fact, this constraint will force the relay stations to accept the use of subcarriers carrying some amount of ICI.

In the following section, a heuristic utility-based resource allocation algorithm is proposed in which the objective functions in (10) and (12) with their constraints are taken into account.

\section{Proposed Resource allocation algorithms}

Taking the above optimization problems into account, the following sections present the proposed resource allocation algorithms.

\subsection{ICI Mitigation through Relay Selection}

This section presents the proposed algorithm in which relay stations will be selected to be the serving RS for certain subcarriers.

Different RSs experience different ICI levels on each subcarrier. Thus, for each subcarrier, it is optimal to select the relay with minimum ICI effects to be the serving RS for that particular subcarrier. By doing so for all subcarriers, the total number of subcarriers $N$ will be divided between the $R$ relay stations. Thus, every RS has a subset of the total number of subcarriers $N$ allocated to it.

Figure 3 illustrates the outcome of the proposed ICI-based relay selection (ICI-RS) compared to random relay selection (R-RS). It can be seen in Figure 3(a) that the proposed relay selection algorithm significantly reduces the average ICI per relay station over the random selection algorithm. In fact, for random selection, the average ICI per RS is reduced due to the random 


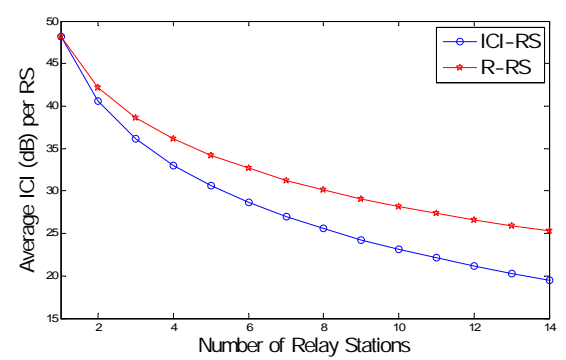

(a) ICI Mitigation / RS

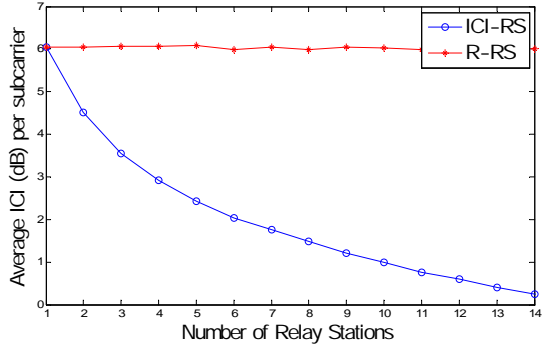

(b) ICI Mitigation / Subcarrier

Figure 3: ICI mitigation using the proposed ICI-based RS selection algorithm

division of the total available ICI between the available relay stations. However, in the proposed algorithm the new relay selection scheme contributes in further minimizing the average ICI per RS by carefully selecting RS with less ICI effects on them.

A relay station $r^{*}$ will be selected to serve a given subcarrier $n$ that satisfies the following condition [15]:

$$
\left(r^{*}\right)=\underset{r}{\arg \min }\left(\sum_{i}^{I}\left(H_{i, r}^{n} L\left(d_{i, r}\right) p_{i}\right)\right), r \in\{\mathcal{R}\}, i \in\{\mathcal{I}\}
$$

Applying this condition on all available subcarriers produces a subset of subcarriers to be used by each relay station. These subcarriers will be allocated to subscribers as described in the following section.

\subsection{Subcarrier Allocation}

At this point, it is assumed that the total power is equally distributed over $N$ subcarriers, to simplify the subcarrier allocation process; that is, $p_{s}^{n}=p_{r}^{n}=P_{T} / N, \forall s, r$. Thus, constraints (11d), (11f) and (11g) can be removed and the optimization problem in (10) is simplified to:

$$
\max \left(U_{R_{T}}\right)
$$


subject to:

$$
\begin{array}{r}
\rho_{s, r}^{n} \in\{0,1\}, \forall n, s, r \\
\sum_{s=1}^{S} \sum_{r=1}^{R} \rho_{s, r}^{n}=1, \forall n \\
\sum_{n=1}^{N} U_{R_{s}^{n}} \geq U_{R_{\min }(s)}, \forall s
\end{array}
$$

A subcarrier allocation algorithm which optimizes the objective function in (15) subject to its constraints (16a)-(16c) is depicted in Algorithm 1.

The urgency, $\mathcal{G}_{s}$ is defined as the necessity of a certain subscriber to be allocated with a subcarrier to meet its minimum rate requirements and is calculated as the difference between the current achieved data rate $\left(R_{s}\right)$ and the minimum data rate requirements $\left(R_{s_{m i n}}\right)$ by each subscriber as follows:

$$
\mathcal{G}_{s}=\left\{R_{s}-R_{s_{\text {min }}}\right\}
$$

The subscriber which minimizes (17) has the highest priority with respect to subcarrier allocation; that is, this subscriber will be allocated the best subcarrier. However, this will allow a certain subscriber who is subject to deep fading over all subcarriers to excessively use most of the available subcarriers, degrading the total achieved rate and disadvantaging the other subscribers. To avoid this problem, the urgency factor is jointly used with the impact of each subcarrier on each subscriber in terms of achievable data rate. This means that the subcarrier will be assigned to subscriber who most urgently needs resources and whose data rate will be significantly increased with the use of this particular subcarrier compared to other subscribers.

This is performed by identifying the subscriber with highest priority $\left(s^{*}\right)$ according to (17). This subscriber will then be able to select the relaysubcarrier set $r^{*}, n^{*}$ by which its instantaneous rate is maximized:

$$
\left(r^{*}, n^{*}\right)=\underset{r, n}{\arg \max }\left(R_{s^{*}, r}^{n}\right), r \in\{\mathcal{R}\}, n \in\left\{\mathcal{N}^{r^{*}}\right\}
$$

Next, the selected relay/subcarrier pair will be used to check the achievable data rate of this pair over the other subscribers, i.e. calculate $R_{s, r^{*}}^{n^{*}}, \forall s \in$ $\mathcal{S}$.

Finally, the negative of the urgency factor $\mathcal{G}_{s}$ in (17) is multiplied by the 
instantaneous rate $R_{s, r^{*}}^{n^{*}}$ to obtain the utility that each subscriber can achieve through the selected relay $\left(r^{*}\right)$ and subcarrier $\left(n^{*}\right)$ :

$$
U_{s, r^{*}}^{n^{*}}=-\mathcal{G}_{s} R_{s, r^{*}}^{n^{*}}
$$

Therefore, the constraint (16c) is now incorporated in the utility function (19), which means that this constraint can be removed and the objective function in (15) modified to:

$$
\max \left(U_{s}\right)
$$

The other constraints $((16 \mathrm{a})$ and $(16 \mathrm{~b}))$ remain the same

This procedure will continue until the rate requirements of all users are achieved; at this point the remaining subcarriers will be allocated to maximize the total sum data rate, and the objective function is similar to (15), but in this case constraint (16c) is omitted. The entire procedure is described in Algorithm 1.

\section{ICI-based water-filling algorithm}

In the previous section, equal distribution of power across subcarriers is considered. However, further performance enhancements may be achieved by distributing the available power resource in a more efficient manner. This section proposes a power allocation algorithm based on the water-filling (WF) approach $[19,16]$.

In OFDMA networks, it is very common to use the WF power allocation algorithm based on the CSI (i.e., subcarriers with superior channel conditions will be allocated more power compared to those with poor channel conditions). However, if the cooperative relays are taken into account, this procedure must be extended to include the CSI between the subscriber and relay stations as well as between the relays and the destination (i.e., two hops), which will increase the algorithm complexity. In spite of the increased complexity, the algorithm is still worth considering due to the potential performance improvement.

Applying the adopted system model, it can be seen that system performance is dominated by the ICI effects on the relay stations. Thus, the level of ICI may be used as an input to the WF algorithm.

In fact, the proposed ICI-based WF algorithm allocates the total power across subcarriers based on the amount of ICI affecting each subcarrier. 


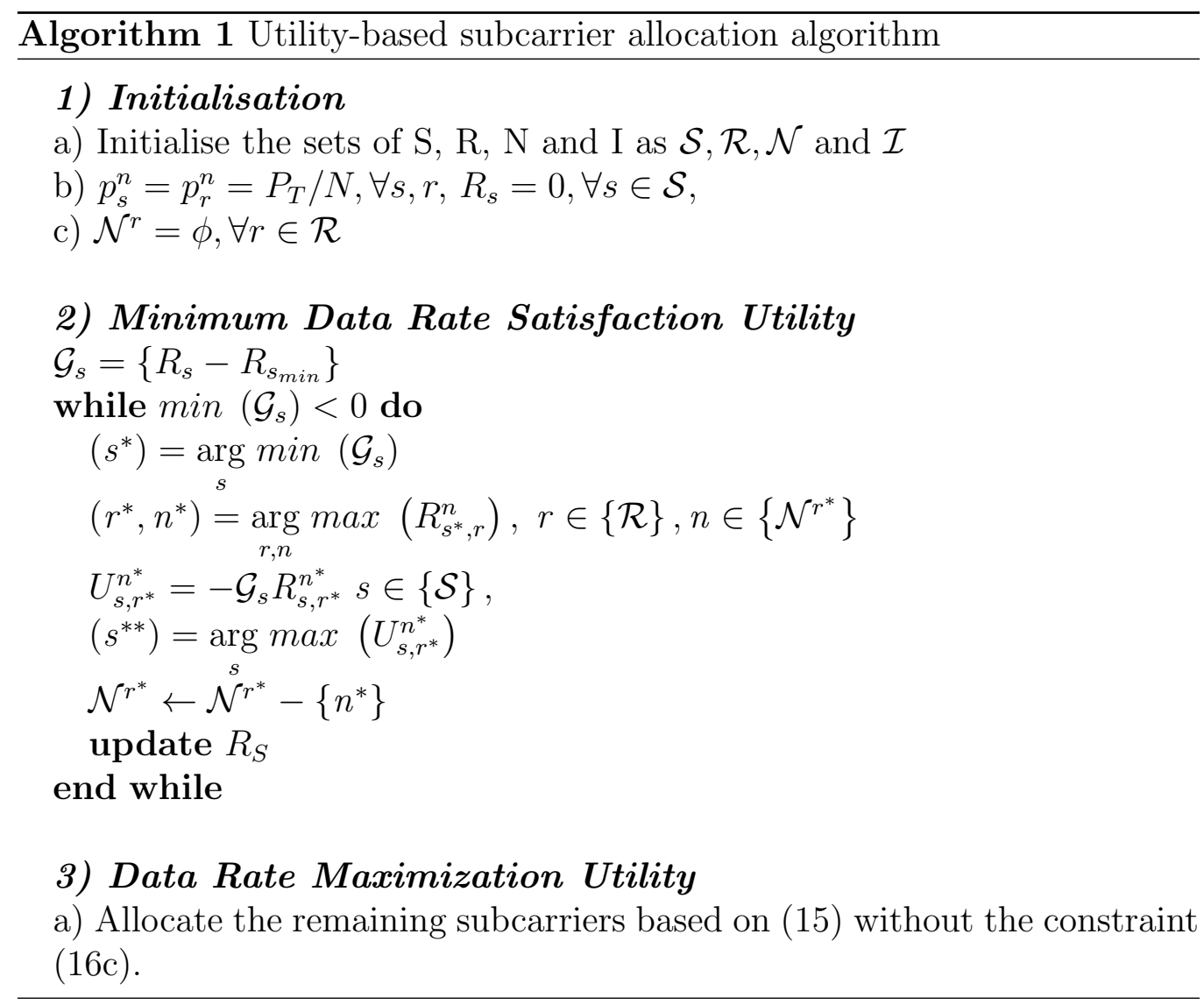




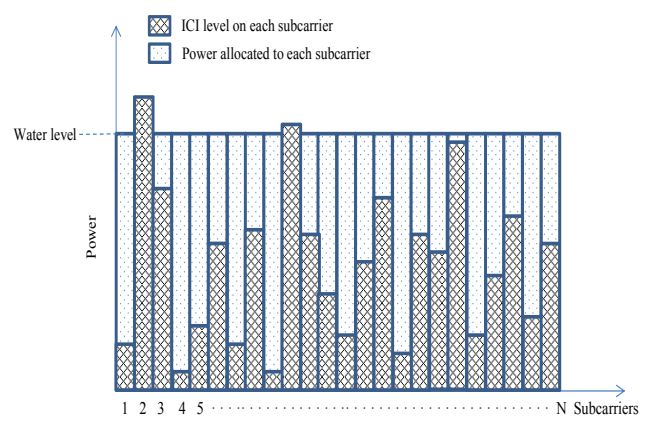

Figure 4: ICI based Water-Filling Algorithm

Therefore, the proposed power allocation scheme only needs to consider the channel between the interfering sources and relay stations (one hop). This assumption is made because the relay stations are located close to the cell edge (i.e., closer to interfering sources of other cells) which means that the amount of the received ICI at relay stations is very high compared to the amount of received ICI at the base station. Hence, the power allocation process is simplified to a level similar to a simple OFDMA network.

As shown in Figure 4, more power is allocated to subcarriers with minimal ICI interference on them (such as; subcarrier number 4), while the ones with relatively high interference are allocated with minimum amount of power (such as subcarrier number 3) or no power at all if the amount of interference exceeds the predetermined water level (such as subcarriers 2 has no power due to high ICI level). With this scheme, the good subcarriers with low ICI are able to get more power and hence utilised efficiently, at the same time, the system avoids using the highly impacted subcarriers by allocating zero power to them (i.e., not using them).

Again, let $I_{I, r}=\sum_{i=1}^{I}\left|h_{i, r}^{n}\right|^{2} L\left(d_{i, r}\right) p_{i}^{n} / \sigma^{2}$, represents the aggregated intercell interference at certain $r^{t h}$ relay station, then the power allocation across subcarriers utilised by that relay station is given by [6]:

$$
p_{r}^{n}=\max \left(\left(\frac{1}{\lambda}-I_{I, r}\right), 0\right)
$$

Where, $\left(\frac{1}{\lambda}\right)$ is chosen such that the power constrain (water level) is satisfied. 
The total available power is derived from (21) and given by:

$$
P_{T}=\sum_{n=1}^{n}\left(\frac{1}{\lambda}-I_{I, r}\right)^{+}
$$

Where, $(x)^{+}=\max (x, 0)$.

As previously discussed, equal power allocation across subcarriers was initially assumed in order to optimize the subcarriers allocation (see algorithm 1). However, once the subcarrier allocation algorithm allocates the subcarriers, the algorithm will proceed to the next step, in which the power across subcarriers is allocated based on the proposed ICI based water-filling power allocation algorithm (21).

\section{Partial Channel state Information Model}

So far, in previous section, it was assumed that the channel state information (CSI) is perfectly known at the resource controller. In reality, perfect knowledge about the CSI requires a huge amount of feedback and cannot be quarantined due to feedback estimation error. However, some statistical information about the CSI could be easily available at the resource controller, this is called partial CSI. In this section, we investigate the proposed allocation performance assuming only partial CSI is available. Equations (2), (3) and (4) describe the SINR rations at the relay station from the direct link and at the destination from the direct and forwarded links respectively. Note here in equations 1-5, the channel gain is assumed to be imperfect due to estimation error, thus the channel gain can be written in terms of estimated value and estimation error as the following:

$$
h_{a, b}=\hat{h}_{a, b}+\epsilon_{a, b}^{n}
$$

where, $h_{a, b}$ is the actual channel gain between nodes $a$ and $b, \hat{h}_{a, b}$ is the estimated channel gain between nodes $a$ and $b$ and $\epsilon_{a, b}^{n}$ is the estimation error between nodes $a$ and $b$ over the $n^{\text {th }}$ subcarrier. In (23) the actual gain (i.e., $\left.h_{a, b}\right)$ is modeled as $\mathcal{C N}\left(\hat{h}_{a, b}, \sigma_{a, b}^{2}\right)$ and its square (i.e., $\left.\left|h_{a, b}\right|^{2}\right)$ follow the noncentral Chi-square distribution with a pdf given as: 


$$
f_{\left|h_{a, b}^{n}\right|^{2}}(\mathcal{V})=\frac{1}{\left(\sigma_{a, b}^{n}\right)^{2}} e^{-\frac{\left(\left|\hat{h}_{a, b}^{n}\right|^{2}+\mathcal{V}\right)}{\left(\sigma_{a, b}^{n}\right)^{2}}} I_{0}\left(2 \sqrt{\frac{\left|\hat{h}_{a, b}^{n}\right|^{2} \mathcal{V}}{\left(\sigma_{a, b}^{n}\right)^{4}}}\right)
$$

Where, $I_{0}$ is the zeroth-order modified Bessel function of the first kind. Assuming that the AWGN effect is small enough to be ignored in (3), then the signal to interference ratio (SIR) at the destination from the direct link is the ratio of two Chi-square distributions given as:

$$
\begin{aligned}
& g(\alpha)=\frac{e^{-\left[\frac{B_{1}^{2}}{2 \sigma_{1}^{2}}+\frac{B_{2}^{2}}{2 \sigma_{2}^{2}}\right]}\left(\frac{1}{4 \sigma_{1}^{2} \sigma_{2}^{2}}\right)}{\left(\frac{\alpha}{2 \sigma_{1}^{2}}+\frac{1}{2 \sigma_{2}^{2}}\right)^{2}} \sum_{j=0}^{\infty}\left(\frac{B_{2}}{2 \sigma_{2}^{2}}\right)^{2 j} \frac{\Gamma(2+j)}{\left(\frac{\alpha}{2 \sigma_{1}^{2}}+\frac{1}{2 \sigma_{2}^{2}}\right)^{j}} \\
& \sum_{i=0}^{j}\left(\frac{2 B_{1} \sigma_{2}^{2}}{2 B_{2} \sigma_{1}^{2}}\right)^{2 i} \frac{\alpha^{i}}{i !(j-i) ! \Gamma(i+1) \Gamma(j-i+1)}
\end{aligned}
$$

Under the same assumptions of zero AWGN, the SIR at the destination from the relay link in (4) can be written as:

$$
S I R_{r, d}^{n}=\frac{p_{s}^{n} g_{r} \mathcal{X}}{p_{i}^{n} g_{r} \mathcal{Y}+g_{r} \mathcal{Z}}
$$

where, $\mathcal{X}$ and $\mathcal{Y}$ are i.i.d random variables follow the pdf given by:

$$
\begin{aligned}
& g(\beta)=2 e^{-\left[\frac{B_{1}^{2}}{2 \sigma_{1}^{2}}+\frac{B_{2}^{2}}{2 \sigma_{2}^{2}}\right]} \times\left(\frac{1}{4 \sigma_{1}^{2} \sigma_{2}^{2}}\right) \sum_{j=0}^{\infty}\left(\frac{\beta \sigma_{2}^{2}}{\sigma_{1}^{2}}\right)^{j / 2} \\
& \left(\frac{B_{2}}{2 \sigma_{2}^{2}}\right)^{2 j} \sum_{i=0}^{j} \frac{\left(\frac{B_{1} \sigma_{2}}{B_{2} \sigma_{1}}\right)^{2 i} N_{2 i-j}\left(2 \sqrt{\frac{\beta}{4 \sigma_{1}^{2} \sigma_{2}^{2} f}}\right)}{i !(j-i) ! \Gamma(i+1) \Gamma(j-i+1)}
\end{aligned}
$$

where $B_{1}$ and $B_{2}$ represents $\left|h_{r, d}^{n}\right|^{2}$ and $\left|h_{s, r}^{n}\right|^{2}$ for the random variable $\mathcal{X}$ and $\left|h_{r, d}^{n}\right|^{2}$ and $\sum_{i=1}^{I}\left|\hat{h}_{i, r}^{n}\right|^{2} L\left(d_{i, r}\right)$ for the random variable $\mathcal{Y} . K_{n}(x)$ is the $n^{\text {th }}$ order modified Bessel function of the second kind [1]. The random variable $\mathcal{Z}$ follows the chi-square distribution given in (24) with a non-centrality pa- 
rameter of $\left|h_{r, d}^{n}\right|^{2}$. Thus, the received SIR at the output of the MRC receiver under the assumption of partial CSI is written as

$$
S I R_{M R C, r}^{n}=\sum_{j=1}^{J} S I R_{j, d}^{n}=\frac{p_{s}^{n} g_{r} \mathcal{X}}{p_{i}^{n} g_{r} \mathcal{Y}+g_{r} \mathcal{Z}}+\mathcal{M}
$$

where $\mathcal{M}$ represents the pdf of the direct SIR which follow the distribution in $(25)$.

\section{Numerical Results}

This section evaluates the system performance of the proposed utilitybased resource allocation algorithms. The achievable aggregate data rate, outage probability and fairness are considered as the main performance metric evaluation. The results were compared to the greedy resource allocation algorithm (in which the subcarriers are selfishly allocated to users with best channel conditions regardless of their data rate requirements [14]) and with the algorithm proposed in [15], which is referred to as the grouping algorithm here.

Matlab software was used to conduct simulations with various parameters. Multiple cells scenario is considered with a single base station located at the center of each cell and four relay stations located around the main base station to serve 20 users who are distributed randomly within the cell. Furthermore, It is assumed that each relay station receives ICI caused by four interfering nodes from neighboring cells. The estimation error $(\epsilon)$ is assumed to be 0.0 for the full CSI case, while in case of partial CSI, $\epsilon=0.01$ or 0.1 . Finally, the SNR ranges from 0 to $30 \mathrm{~dB}$, and the received ICI is calculated as a function of interfering node's transmit power and it's distance to the receiving node.

Figure 5 shows the total achieved sum data rate of the proposed resource allocation algorithm compared to the greedy and grouping algorithms. As expected, the total sum rate increases with SNR in all cases. However, the greedy algorithm outperforms the proposed and grouping algorithms in terms of total sum rate. This is expected because the greedy algorithm is 'selfish' and therefore results in optimal performance with respect to total sum rate. However, the proposed utility-based algorithm provides a significant improvement over the grouping algorithm and achieves a total sum data rate which approaches that of the greedy algorithm. This is because in the proposed 


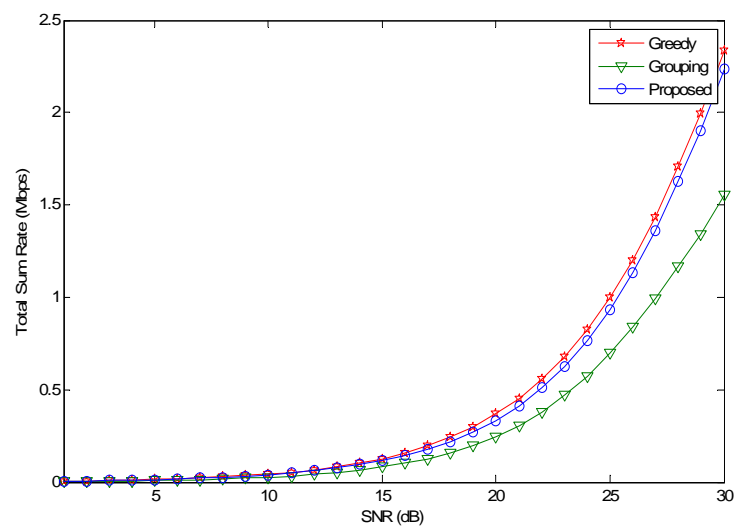

Figure 5: Total sum data rate as a function of SNR; $N=256, I=4, R=4$ and $S=20$

algorithm, the current minimum data rate requirements of every user and the impact of the allocation of every subcarrier on the total capacity are jointly considered to dynamically update the selection priority, while in the grouping algorithm the priority is always given based on users urgency only.

On the other hand, Figure 6 shows the performance of the same three algorithms in terms of outage probability. Since the greedy algorithm is selfish, it does not take the data rate requirements into account; therefore, it has the worst performance in terms of outage probability. The proposed algorithm significantly outperforms the grouping and greedy algorithms in terms of outage probability. The low performance of the grouping algorithm compared to the proposed algorithm is caused by the excessively use of available subcarriers by subscribers who are subject to deep fading over all subcarriers. Thus, other users are disadvantaged and are unable to meet their minimum rate requirements.

The three algorithms were also evaluated in terms of total sum data rate versus the number of ICI sources as shown in Figure 7. The figure shows that the proposed algorithm significantly outperforms the grouping algorithm for all levels of ICI, although it has slightly lower performance compared to the greedy algorithm.

Jain's fairness index (FI) [8] has been widely adopted to test the fairness capabilities of resource allocation algorithms. Based on this index, an algorithm that allocates similar average data rates to all users is considered to be fair. However, in a situation when there are different data rate requirements for subscribers, this definition of fairness becomes inappropriate. Therefore, 


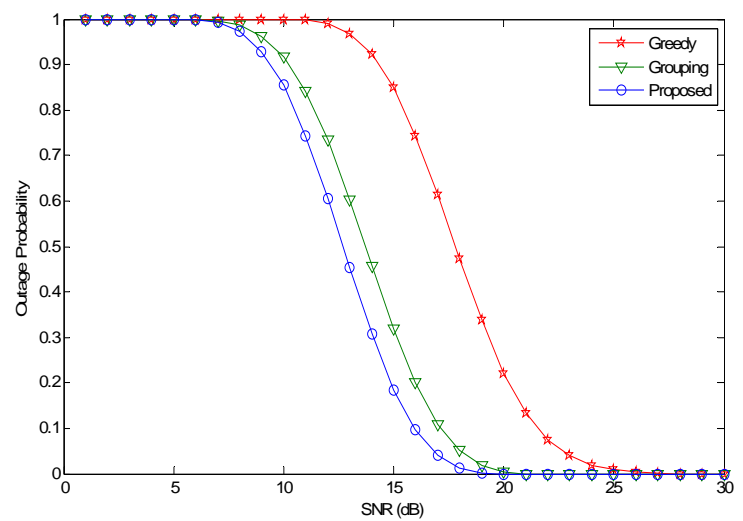

Figure 6: Outage probability as a function of SNR; $N=256, I=4, R=4$ and $S=20$

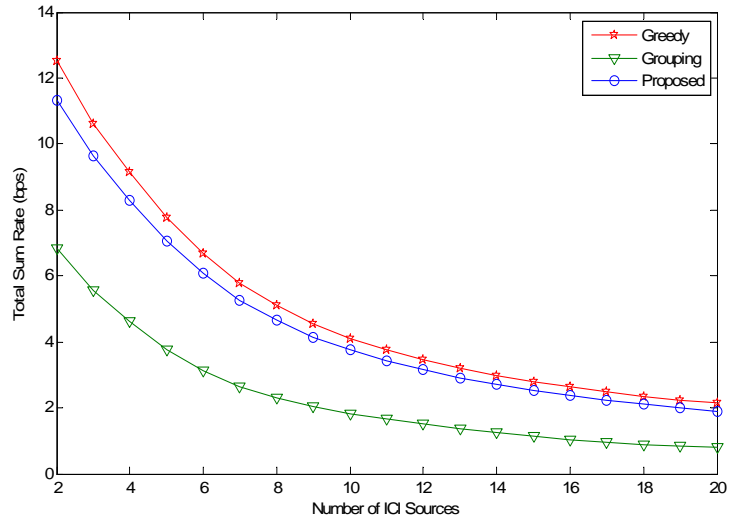

Figure 7: Total sum data rate as a function of number of ICI sources; $N=256, R=6$ and $S=10$ 


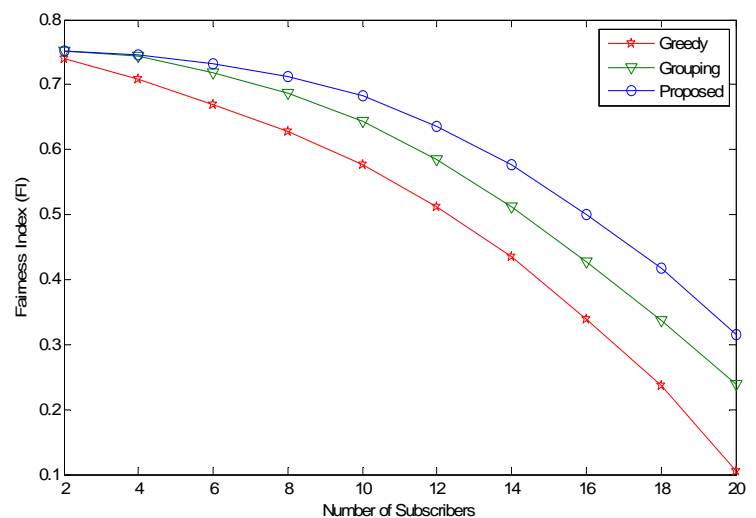

Figure 8: Fairness index as a function of number of subscribers, $N=256, I=4$ and $R=4$

in this paper we have modified Jain's FI by incorporating the minimum data rate requirements $\left(R_{s_{\text {min }}}\right)$ into the formula as follows: $F I=\frac{\left(\sum_{s=1}^{S} \alpha_{s}\right)^{2}}{S \sum_{s=1}^{S} \alpha_{s}^{2}}$, where, $\alpha_{s}=\frac{\bar{R}_{s}}{R_{s_{\min }}}, \bar{R}_{s}$ is the $s^{\text {th }}$ subscriber's average achieved data rate. $F I$ takes a value between ' 0 ' and '1', and the higher $F I$ value indicates that the system is fairer.

Figure 8 depicts the fairness performance of the aforementioned three algorithms. It can be seen that the proposed utility-based algorithm achieves the highest performance in terms of fairness compared to the other two with various number of subscribers. This indicates that the proposed algorithm results in a fair balance between the achieved data rates and outage taking the individual's minimum data rate requirements into account. As the number of subscribers increases, it becomes harder to maintain the same level of fairness in all algorithms.

From Figures 5, 7 and 8, it can be seen that he proposed algorithm lies between the other two algorithms (greedy and grouping), by which the resource channels are efficiently allocated (when compared to grouping algorithm) without compromising the fairness between users (like the greedy algorithm).

The preceding results assume that power allocation is equal across all subcarriers. By contrast, the performance of the proposed interference-based water filling power allocation algorithm is evaluated in terms of total sum data rate versus SNR in Figure 9. The proposed utility-based subcarrier allocation was utilised with both equal-power and ICI-based power allocation 


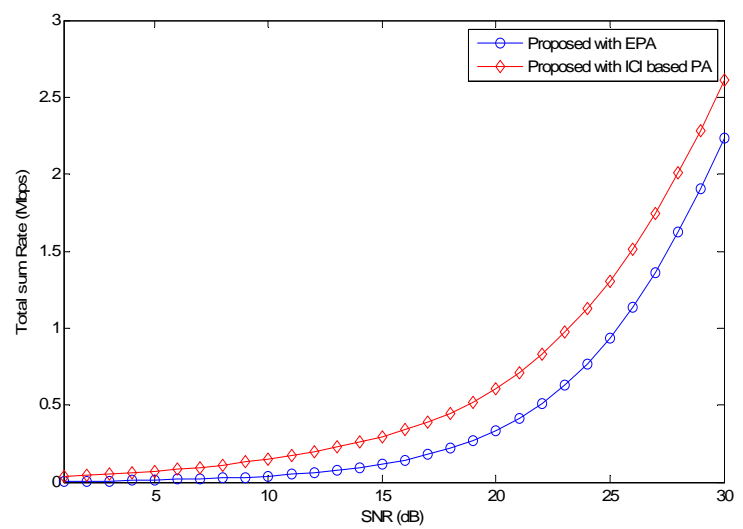

Figure 9: Total sum data rate as a function of SNR, $N=256, I=4, R=4$ and $S=20$

algorithms. It can be seen that the proposed ICI-based water filling approach improves the total sum data rate compared to the equal power allocation algorithm.

Figure 10 depicts the system performance in terms of total sum data rates considering full availability of CSI as well as partial channel state information (PCSI).

In this figure, the system performance was predicted assuming that the estimation error $(\epsilon)$ is zero, hence the available CSI are full and accurate. Then, the actual achieved system performance was obtained assuming that the estimation error is larger than zero (i.e., $\epsilon=0.01$ and 0.1 ) hence the available CSI are partial.

It can be seen from the figure that the system performance when considering full CSI is in fact inaccurate. This is expected because the estimation error was not taken into account during the resource allocation process, therefore the allocation outcome is not efficient for this network. The figure also shows that the actual performance is less than the predicted performance when taking PCSI into account with some estimation error. In spite of the performance reduction based on PCSI compared to the predicted performance based on full CSI, the fact that the system performance based on PCSI reflects the actual achieved performance cannot be ignored. Therefore, it is worth considering a PCSI in order to obtain accurate results, hence more efficient resource allocation can be designed.

Furthermore, it can be seen that the performance is reduced as the estimation error value increases. This is because the resource allocation depends 


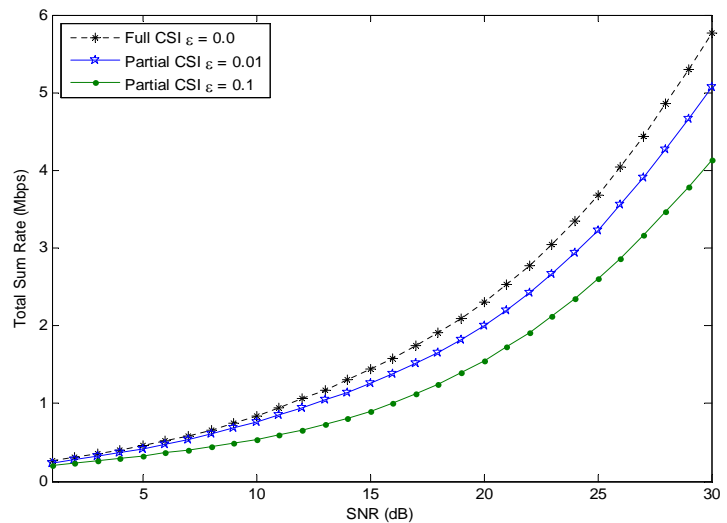

Figure 10: Total achieved sum rate of the proposed algorithm with Full and partial CSI, $\mathrm{S}=20, \mathrm{~N}=256, \mathrm{R}=4$ and $\epsilon=0,0.01$ and 0.1

highly on the available channel information; therefore, the algorithm becomes less efficient when the channel information is estimated with high estimation error.

\section{Conclusion}

This paper proposes a utility-based resource allocation algorithm which takes the ICI in a multiple cell environment into account. The results show that the proposed utility-based algorithm outperforms the grouping algorithm in terms of total achievable data rate, outage probability and fairness. On the other hand, the proposed algorithm achieves an aggregate data rate which is slightly lower than that of the greedy algorithm. However, the proposed algorithm significantly reduces the outage probability and enhances the fairness compared to the greedy algorithm. Furthermore, the proposed ICI-based WF algorithm further enhances the total sum data rate of the proposed algorithm. In addition to that, partial channel state information (PCSI) model was introduced and considered in this paper, the result with respect to PCSI shows that an accurate prediction of the achievable data rate can be obtained when partial CSI is considered

[1] Irena A. (editor) Abramowitz, Milton (editor); Stegun. Handbook of mathematical functions with formulas, graphs, and mathematical tables. 
U.S. Department of Commerce, National Bureau of Standards, USA, 1964.

[2] Mohammed Al-Imari, Pei Xiao, MuhammadAli Imran, and Rahim Tafazolli. Low complexity subcarrier and power allocation algorithm for uplink ofdma systems. In EURASIP Journal on Wireless Communications and Networking, Springer International Publishing AG, volume 2013, 2013. doi: 10.1186/1687-1499-2013-98.

[3] M.K. Awad and Xuemin Shen. Ofdma based two-hop cooperative relay network resources allocation. In Communications, 2008. ICC '08. IEEE International Conference on, pages 4414-4418, May 2008. doi: 10.1109/ICC.2008.828.

[4] M. Castaneda, M.T. Ivrlac, J.A. Nossek, I. Viering, and A. Klein. On downlink intercell interference in a cellular system. In Personal, Indoor and Mobile Radio Communications, 200\%. PIMRC $200 \%$. IEEE 18th International Symposium on, pages 1-5, Sept 2007. doi: 10.1109/PIMRC.2007.4394052.

[5] Y.-H. Chung and C.-J. Chang. A balanced resource scheduling scheme with adaptive priority thresholds for ofdma downlink systems. Vehicular Technology, IEEE Transactions on, 61(3):1276 -1286, march 2012. ISSN 0018-9545. doi: 10.1109/TVT.2012.2186832.

[6] Thomas M. Cover and Joy A. Thomas. Elements of Information Theory. Wiley-Interscience, New York, NY, USA, 1991. ISBN 0-471-06259-6.

[7] Kun Dong, Hui Tian, Xingmin Li, and Qiaoyun Sun. A distributed inter-cell interference coordination scheme in downlink multicell ofdma systems. In Consumer Communications and Networking Conference (CCNC), 2010 7th IEEE, pages 1 -5, jan. 2010. doi: 10.1109/CCNC.2010.5421833.

[8] Rajendra K. Jain, Dah-Ming W. Chiu, and William R. Hawe. A Quantitative Measure Of Fairness And Discrimination For Resource Allocation In Shared Computer Systems. Technical report, Digital Equipment Corporation, September 1984.

[9] Hyundoo Jeong, Jae Hong Lee, and Hanbyul Seo. Resource allocation for uplink multiuser ofdm relay networks with fairness constraints. In 
Vehicular Technology Conference, 2009. VTC Spring 2009. IEEE 69th, pages 1-5, April 2009. doi: 10.1109/VETECS.2009.5073835.

[10] I. Krikidis, J. Thompson, S. Mclaughlin, and N. Goertz. Max-min relay selection for legacy amplify-and-forward systems with interference. Wireless Communications, IEEE Transactions on, 8(6):3016-3027, June 2009. ISSN 1536-1276. doi: 10.1109/TWC.2009.080383.

[11] Wen-Hsing Kuo and Wanjiun Liao. Utility-based resource allocation in wireless networks. Wireless Communications, IEEE Transactions on, 6(10):3600-3606, October 2007. ISSN 1536-1276. doi: 10.1109/TWC.2007.05942.

[12] Wen-Hsing Kuo and Wanjiun Liao. Utility-based resource allocation in wireless networks. Wireless Communications, IEEE Transactions on, 6(10):3600 -3606, october 2007. ISSN 1536-1276. doi: 10.1109/TWC.2007.05942.

[13] Chang Liu, Sihai Zhang, Xiaowei Qin, and Wuyang Zhou. Utilitybased resource allocation in ofdma relay networks with service differentiation. In Wireless Communications and Networking Conference (WCNC), 2011 IEEE, pages 72 -77, march 2011. doi: 10.1109/WCNC.2011.5779109.

[14] S. Najeh, H. Besbes, and A. Bouallegue. Greedy algorithm for dynamic resource allocation in downlink of ofdma system. In Wireless Communication Systems, 2005. 2nd International Symposium on, pages 475 -479, sept. 2005. doi: 10.1109/ISWCS.2005.1547746.

[15] Nidhal Odeh, Mehran Abolhasan, and Farzad Safaei. Low complexity interference aware distributed resource allocation for multi-cell OFDMA cooperative relay networks. In IEEE Wireless Communications and Networking Conference (IEEE WCNC 2010 - MAC), Sydney, Australia, 4 2010.

[16] Q. Qi, A. Minturn, and Y. Yang. An efficient water-filling algorithm for power allocation in ofdm-based cognitive radio systems. In Systems and Informatics (ICSAI), 2012 International Conference on, pages 20692073, May 2012. doi: 10.1109/ICSAI.2012.6223460. 
[17] Yanyan Shen, Gang Feng, Bo Yang, and Xinping Guan. Resource allocation with proportional rate fairness in orthogonal frequency division multiple access relay networks. Wireless Comm. and Mobile Computing, 2012. ISSN 1530-8677.

[18] Zhenhui Shen, Xiaoxiang Wang, and Hongtao Zhang. Power allocation and subcarrier pairing for ofdm-based af cooperative diversity systems. In Vehicular Technology Conference, 2009. VTC Spring 2009. IEEE 69th, pages 1-5, April 2009. doi: 10.1109/VETECS.2009.5073808.

[19] H. Wang, Q. Cui, X. Tao, M. Valkama, and Y. J. Guo. Optimal cooperative water-filling power allocation for ofdm system. In Wireless Communications and Networking Conference (WCNC), 2013 IEEE, pages 3742-3747, April 2013. doi: 10.1109/WCNC.2013.6555170.

[20] Chih-Ming Yen, Chung-Ju Chang, and Li-Chun Wang. A utility-based tmcr scheduling scheme for downlink multiuser mimo-ofdma systems. Vehicular Technology, IEEE Transactions on, 59(8):4105 -4115, oct. 2010. ISSN 0018-9545. doi: 10.1109/TVT.2010.2062546.

[21] Danhua Zhang, Youzheng Wang, and Jianhua Lu. Qos aware relay selection and subcarrier allocation in cooperative ofdma systems. Communications Letters, IEEE, 14(4):294 -296, april 2010. ISSN 1089-7798.

[22] Danhua Zhang, Xiaoming Tao, Jianhua Lu, and Meng Wang. Dynamic resource allocation for real-time services in cooperative ofdma systems. Communications Letters, IEEE, 15(5):497 -499, may 2011. ISSN 10897798. doi: 10.1109/LCOMM.2011.031411.101843.

[23] H. Zhang, C. Jiang, N. C. Beaulieu, X. Chu, X. Wang, and T. Q. S. Quek. Resource allocation for cognitive small cell networks: A cooperative bargaining game theoretic approach. IEEE Transactions on Wireless Communications, 14(6):3481-3493, June 2015. ISSN 1536-1276.

[24] H. Zhang, H. Xing, J. Cheng, A. Nallanathan, and V. Leung. Secure resource allocation for ofdma two-way relay wireless sensor networks without and with cooperative jamming. IEEE Transactions on Industrial Informatics, PP(99):1-1, 2015. ISSN 1551-3203.

[25] H. Zhang, C. Jiang, X. Mao, and H. H. Chen. Interference-limited resource optimization in cognitive femtocells with fairness and imperfect 
spectrum sensing. IEEE Transactions on Vehicular Technology, 65(3): 1761-1771, March 2016. ISSN 0018-9545.

[26] Haijun Zhang, Chunxiao Jiang, N. C. Beaulieu, Xiaoli Chu, Xiangming Wen, and Meixia Tao. Resource allocation in spectrum-sharing ofdma femtocells with heterogeneous services. IEEE Transactions on Communications, 62(7):2366-2377, July 2014. ISSN 0090-6778.

[27] Yan Zhu and Haitao Zheng. Understanding the impact of interference on collaborative relays. Mobile Computing, IEEE Transactions on, 7(6): 724-736, June 2008. ISSN 1536-1233. doi: 10.1109/TMC.2007.70790. 